\title{
SOX4 interacts with plakoglobin in a Wnt3a- dependent manner in prostate cancer cells
}

\author{
Yu-Heng Lai ${ }^{1,2}$, Jessica Cheng ${ }^{2}$, Dongmei Cheng ${ }^{2}$, Mattie E Feasel ${ }^{2}$, Kyle D Beste ${ }^{2}$, Junmin Peng ${ }^{3,4,5,6}$, Asma Nusrat $^{2}$ \\ and Carlos S Moreno ${ }^{2,4^{*}}$
}

\begin{abstract}
Background: SOX4 is a developmental transcription factor that is required for differentiation and proliferation in multiple tissues. SOX4 is overexpressed in many human malignancies, but the precise role of SOX4 in cancer progression is still not well understood. Thus, the identification of additional SOX4 binding partners is essential for elucidating the mechanism of SOX4-mediated effects in cancer progression.
\end{abstract}

Results: Here, we have adapted a one-step affinity purification method that enables rapid purification of SOX4 complexes via intracellular biotinylation of the amino-terminus of SOX4 to perform large-scale proteomics analysis. We have discovered that junction plakoglobin (JUP) interacts with SOX4 in both the cytosol and the nucleus and the interaction between SOX4 and plakoglobin is significantly increased when prostate and breast cancer cells are stimulated with WNT3A. Interactions between SOX4 and plakoglobin were further enhanced by the nuclear export inhibitor leptomycin B (LMB), suggesting that plakoglobin promotes nuclear export of SOX4. The SOX4-plakoglobin complex affected the expression of Wnt pathway target genes and SOX4 downstream targets, such as AXIN2, DICER1, and DHX9. In addition, SOX4 DNA binding activity to the promoters of DICER1, AXIN2, DHX9 and SOX4 itself was reduced by conditions that promote SOX4-plakoglobin complex formation. Conditions that enhanced SOX4plakoglobin interactions resulted in reduced transcriptional activity of $\beta$-catenin luciferase reporters.

Conclusions: These data suggest that this newly identified interaction between SOX4 and plakoglobin is inhibitory and provides new insights into the role of SOX4 in key pathways in cell proliferation, development, and cancer progression.

\section{Background}

The sex-determining region Y (SRY) box, or SOX, family consists of 20 highly conserved transcription factors in humans that play important roles in development [1]. SOX4 is a $47-\mathrm{kDa}$ protein that is encoded by a single exon gene and contains a conserved signature sequence in the high-mobility group (HMG) DNA-binding domain (DBD) related to the TCF/LEF family of transcription factors. The key effector of the canonical Wnt pathway, $\beta$-catenin, forms complexes with TCF/ LEF HMG box factors and stimulates the transcription of Wnt downstream targets. Many studies have shown that $\beta$-catenin is regulated at several levels in cancer progression, but less is known about the regulation of

\footnotetext{
* Correspondence: cmoreno@emory.edu

${ }^{2}$ Department of Pathology \& Laboratory Medicine, Emory University School of Medicine, Atlanta, GA 30322, USA

Full list of author information is available at the end of the article
}

the TCF/LEF transcription factor family. Structurally related to TCF/LEFs, several members of the SOX family, SOX17, SOX7, SOX9, and SOX4 have been implicated in regulating $\beta$-catenin activity [2-4]. Gainand loss-of-function analyses have demonstrated that SOX17 and SOX7 proteins antagonize the Wnt pathway by competing with TCFs for $\beta$-catenin binding while SOX4 may function to stabilize $\beta$-catenin protein to help activate expression of target genes and promote cellular proliferation [5]. Although these findings have implicated how SOX proteins regulate the transcriptional output of Wnt pathway, the precise role of SOX4 in the Wnt pathway remains unclear.

In humans, tight regulation of the levels of transcriptional factors is crucial to maintain tissue homoeostasis and many have been found to be oncogenic when their expression is deregulated or when their activity is functionally altered [6]. For example, SOX4 is overexpressed
C Biomed Central

() 2011 Lai et al; licensee BioMed Central Ltd. This is an Open Access article distributed under the terms of the Creative Commons Attribution License (http://creativecommons.org/licenses/by/2.0), which permits unrestricted use, distribution, and reproduction in any medium, provided the original work is properly cited. 
in several cancers, such as bladder cancer, in which it is 5-fold upregulated compared with normal tissues [7]. SOX4 is also upregulated at the mRNA and protein level in prostate cancer and this upregulation is correlated with higher Gleason score or tumor grade [8]. In addition, SOX4 is overexpressed in leukemias, melanomas, glioblastomas, medulloblastomas [9], and lung cancer [10]. SOX4 is also overexpressed in endometrial cancer through methylation-mediated silencing of miR129-2 [11]. Consistent with the concept that SOX4 is an oncogene, three independent studies searching for oncogenes have found SOX4 to be one of the most common retroviral integration sites, resulting in increased mRNA [12-14].

Junction plakoglobin (JUP), also known as $\gamma$-catenin, is a major component of the submembrane of adherens junctions and desmosomes in mammalian cells [15]. It is closely related to the Drosophila segment polarity gene armadillo, which has a role in the transduction of transmembrane signals that regulate cell fate [16,17]. Plakoglobin shares more than $76 \%$ homology with $\beta$ catenin, contains a central armadillo repeat domain flanked by the carboxyl and amino terminal domains, and functions in cell-cell junctions, along with $\beta$-catenin and $\alpha$-catenin when coupled with cadherins [18]. While it is known that $\beta$-catenin is essential in the Wnt signaling cascade, plakoglobin also binds to TCF/LEF factors, and has lower TCF/LEF-dependent transcriptional activity compared to $\beta$-catenin when endogenous $\beta$-catenin is depleted $[19,20]$.

Mass spectrometry is a highly sensitive technique that enables the rapid identification of proteins and also protein-protein interactions from a variety of biological samples. When combined with affinity purification, whole or targeted protein interaction networks can be elucidated [21]. To investigate the cellular functions of SOX4, we have developed a one-step affinity purification method that enables rapid purification of SOX4 complexes in LNCaP cells, a prostate cancer cell line. Here, in this study, we identified proteins that interact with SOX4 in LNCaP cells by liquid chromatography-tandem mass spectrometry (LC-MS/MS), including plakoglobin. This novel interaction between SOX4 and plakoglobin may provide insights into the role of SOX4 in key pathways in cell proliferation, development, and cancer progression.

\section{Methods}

\section{Reagents and cell culture}

LNCaP, PC3M, and MDA-MB-231 cells were cultured as described [22] by American Type Culture Collection except using T-medium (Invitrogen) for LNCaP cells. HA-tagged SOX4 was cloned into pHR-UBQ-IRESeYFP- $\triangle \mathrm{U} 3$ lentiviral vector (gift from Dr. Hihn Ly,
Emory University), and stable cells were isolated, as previously described [23]. Recombinant WNT3A was purchased from R \& D Systems (5036-WNP) and reconstituted in $0.1 \% \mathrm{BSA} / \mathrm{PBS}$ prior to use. Nuclear export inhinbitor leptomycin b (LMB) was purchased from Sigma (L2913). Cells were treated for 24 hrs with $100 \mathrm{ng} / \mathrm{ml}$ WNT3A, $20 \mu \mathrm{M}$ LMB, or both unless otherwise noted.

\section{Biotinlyated HA-tagged SOX4 expression construct}

The pREP4-BLRPwt-IRES-BirA-XL9 plasmid was a gift from Dr. Jeremy Boss (Emory University). The pcDNA3.1-HisA-HASOX4 was constructed as described [8]. To generate pREP4-BLRPwt-HASOX4-IRES-BirAXL9, HA-tagged SOX4 from pcDNA3.1-HisA-HASOX4 was excised at KpnI and XbaI sites and overhangs were filled in with Klenow fragment. Filled-in HA-SOX4 was then blunt ligated into the filled-in NotI site of pREP4BLRPwt-IRES-BirA-XL9.

\section{Purification of biotinlyated HA-tagged SOX4}

The pREP4-BLRPwt-HASOX4-IRES-BirA-XL9 and control empty vector were transfected into two $90 \%$ confluent $100 \mathrm{~mm}$ dishes of LNCaP cells respectively. Fortyeight hours post-transfection, the plates were then placed on ice and the cells were washed twice with icecold PBS, and lysed in $1 \mathrm{ml} /$ plate with ice-cold IP lysis buffer $(0.137 \mathrm{M} \mathrm{NaCl}, 0.02 \mathrm{M}$ Tris pH8.0, 10\% glycerol, $1 \%$ NP-40) supplemented with protease inhibitors, and harvested by scraping. Biotinlyated HA-tagged SOX4 complexes were purified by incubating with 50 ul slurry of Dynabeads ${ }^{\circledR}$ M280 Streptavidin (Invitrogen) at $4^{\circ} \mathrm{C}$ for 2 hrs. The beads ware washed 3 times with IP lysis buffer and eluted by boiling in Laemmli sample buffer.

\section{Sample preparation for mass spectrometry}

Five percent of the proteins resulting from the purification were subject to $4-15 \%$ gradient SDS-PAGE and silver staining to analyze sample purity. The rest of the purified proteins were subjected to $4-15 \%$ gradient SDSPAGE and concentrated on a very short distance $(\sim 2$ $\mathrm{mm}$ long), and the protein in those bands were excised for in-gel digestion and Liquid chromatography coupled with tandem mass spectrometry based on an optimized protocol [21].

\section{Co-immunoprecipitation and Western blot}

Cells were washed twice with ice-cold PBS, lysed in 1 $\mathrm{ml} /$ plate with ice-cold IP lysis buffer supplemented with protease inhibitors, and harvested by scraping. The whole cell lysates were pre-cleared and then incubated with $25 \mathrm{ul}$ slurry of Dynabeads ${ }^{\circledR}$ M280 Streptavidin or protein $\mathrm{G}$ (Invitrogen) at $4^{\circ} \mathrm{C}$ for $2 \mathrm{hrs}$. The beads were washed 3 times with IP lysis buffer and eluted by boiling 
in Laemmli sample buffer before running SDS-PAGE. Western blots were performed as previously described [23]. Antibodies to plakoglobin (13-8500, Invitrogen) and hemagglutinin (HA12CA5) were used in immunoprecipitations (IPs). Antibodies to plakoglobin (610253, BD Biosciences), SOX4 (LS-B3520, LifeSpan Biosciences), $\beta$-actin (3700S, Cell Signaling), and HA $16 B 12$ (AFC-101P-1000, Covance Research Products) were used in Western blot.

\section{Confocal microscopy}

Sub-confluent LNCaP HASOX4 stable cells were grown on glass cover slips, and serum starved for $24 \mathrm{hrs}$ with $0.5 \%$ FBS (fetal bovine serum) T-Medium before treating with $100 \mathrm{ng} / \mathrm{ml}$ WNT3A, $20 \mu \mathrm{M} \mathrm{LMB}$, or both for another $24 \mathrm{hrs}$. Cells were washed three times with Hank's Balanced Salt Solution $\left(\mathrm{HBSS}^{+}\right)$and fixed with $3.7 \%$ paraformaldehyde for $20 \mathrm{~min}$ at room temperature. Cells were permeablized with $100 \%$ ethanol for 20 min at

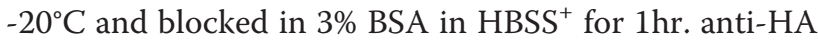
16B12 (1:500 dilution) and plakoglobin antibodies (1:50 dilution) (sc-H80, Santa Cruz Biotechnology) were used as primary antibodies and were diluted in blocking buffer and incubated for $1 \mathrm{hr}$. Then cells were washed in $\mathrm{HBSS}^{+}$ and incubated in fluorescence-labeled secondary antibodies for $1 \mathrm{hr}$ at room temperature. Cells were washed in $\mathrm{HBSS}^{+}$and then stained with Topro (T3605, Invitrogen) for $5 \mathrm{~min}$ at room temperature. Labeled cells were then washed in $\mathrm{HBSS}^{+}$and mounted in p-phenylene diamine antifade agent. Confocal fluorescence images were captured using a laser-scanning microscope.

\section{Cell fractionation into nuclear and cytosolic lysates}

One 100-mm dish of LNCaP HASOX4 stable cells was grown to $80-90 \%$ confluency and serum starved for 24 hrs with $0.5 \%$ FBS T-Medium before treating with 100 $\mathrm{ng} / \mathrm{ml}$ WNT3A, $20 \mu \mathrm{M}$ LMB, or both for another 24 hrs. Crude subcellular fractionation was performed as previously described using digitonin, NP40, and RIPA lysis methods [24].

\section{Quantitative real-time PCR}

Ninety-percent confluent cells were harvested using the RNeasy kit (Qiagen), and reverse transcription was performed using iScript cDNA Synthesis Kit (Bio-Rad Laboratories). Quantitative real-time PCR (qPCR) was performed using iQ SYBR Green Supermix (Bio-Rad Laboratories) on a Bio-Rad iCycler using $18 \mathrm{~s}$ or $\beta$-actin as a control, and data were analyzed using the $\delta \mathrm{Ct}$ method [25].

\section{Chromatin immunoprecipitation (ChIP) assay}

One 100-mm dish of LNCaP HASOX4 stable cells was grown to $80-90 \%$ confluency. Cells were fixed with $1 \%$ formaldehyde, then lysed, and sonicated as described [26]. Sonicated chromatin was precleared and then immunoprecipitated with $4 \mu \mathrm{g}$ of anti-HA 12CA5 ascites or mouse IgG overnight and immunoprecipitated by Dynabeads ${ }^{\circledR}$ Protein G (10004D, Invitrogen) for $2 \mathrm{hrs}$ at $4^{\circ} \mathrm{C}$. Beads were washed and eluted as described [26]. ChIP DNA was purified and then subjected to PCR amplification.

\section{Luciferase reporter assay}

Cells grown in twelve-well tissue culture plates were transfected with $0.5 \mu \mathrm{g}$ of either TOP-flash or FOP-flash with $0.04 \mu \mathrm{g}$ of TK-Renilla control vector (Promega). At $6 \mathrm{hrs}$ post-transfection, cells were placed in $0.5 \% \mathrm{FBS}$ medium for recovery and serum starvation. At $24 \mathrm{hrs}$ post-transfection, cells were treated with WNT3A and LMB, or both. Reporter gene activity was measured in a TD-20/20 luminometer (Turner Design) with the DLR Luciferase Assay System (Promega) after $48 \mathrm{hrs}$ and was normalized for transfection efficiency by measuring Renilla luciferase activity.

\section{siRNA transfection}

The siRNA sequence for plakoglobin AGTCGGCCATTGTGCATCT was targeted at the 5' end of the gene lacking of homology with other catenin members [27] (Dharmacon RNA Technologies). LNCaP HASOX4 cells were transfected using Lipofectamine 2000 (Invitrogen) with plakoglobin or scramble siRNAs at final concentration of $200 \mathrm{nM}$. At 6 hrs post-transfection, cells were placed in $0.5 \%$ FBS medium for recovery and serum starvation. At 24 hrs post-transfection, cells were treated with WNT3A, LMB, or both for Western blot analysis or chromatin immunoprecipitation assay.

\section{Results}

\section{Identification of proteins that interact with SOX4}

To identify proteins that stably interact with SOX4, we developed a one-step affinity purification method that allows for rapid purification of SOX4 complexes. The pREP4-BLRPwt-IRES-BirA-XL9 plasmid contains the birA gene of $E$. coli that encodes a biotin holoenzyme synthetase [28]. We cloned the human SOX4 gene into this vector to generate an amino-terminal fusion to a BirA recognition sequence to produce transiently expressed, intracellularly biotinylated SOX4 protein in $\mathrm{LNCaP}$ prostate cancer cells, and purified SOX4 complexes with streptavidin-linked magnetic beads to perform large scale proteomics analysis. Protein quantity and purity were checked by SDS-PAGE analysis and silver staining prior to mass spectrometry analysis (Figure 1A).

\section{Plakoglobin interacts with SOX4}

LC-MS/MS analysis identified junction plakoglobin (JUP) as a SOX4 binding protein via two trypsinized fragments 


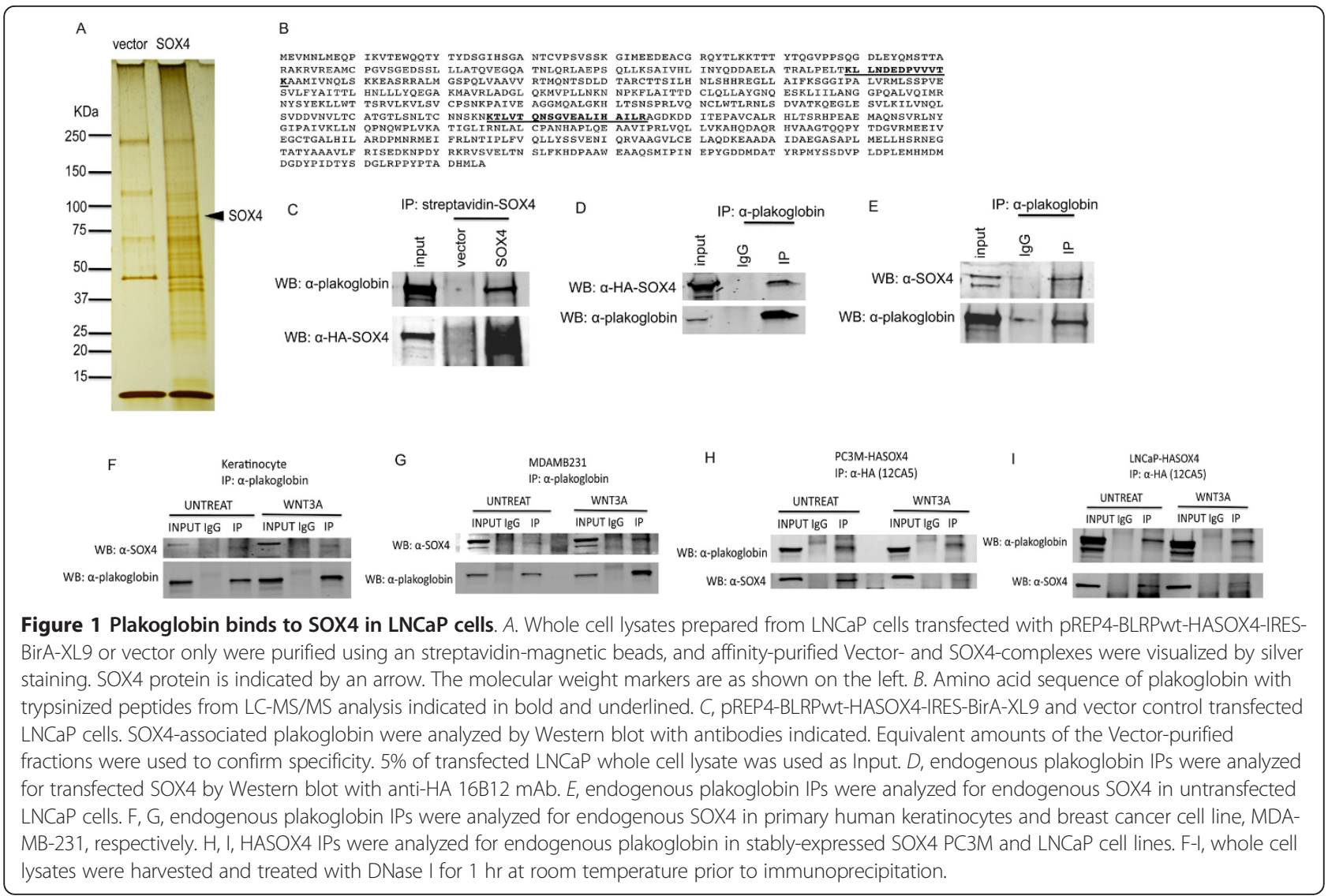

that perfectly matched to human plakoglobin sequences (Figure 1B). To confirm this interaction, we first repeated the transient transfection of the pREP4-BLRPwt-IRESBirA-XL9-HASOX4 or vector control plasmid into LNCaP cells, and performed streptavidin-magnetic bead based purification followed by anti-plakoglobin (JUP) immunoblotting. As expected, the result of the immunoprecipitation (IP) and western blot validated the interaction between SOX4 and plakoglobin (Figure 1C). Furthermore, we performed a reverse-IP immunoprecipitating endogenous plakoglobin and probing for transfected HA-SOX4 (Figure 1D) and endogenous SOX4 in untransfected LNCaP cells (Figure 1E). To determine that the interactions were not cell-line specific or DNA-dependent, we repeated the immunoprecipitations in primary human keratinocytes, MDA-MB-231 breast cancer cells, and PC3M prostate cancer cells (Figure 1F-I) following DNase I digestion of whole cell lysates. Taken together, these data demonstrate that SOX4 directly interacts with plakoglobin and that plakoglobin is a novel SOX4 binding partner.

Interaction between SOX4 and plakoglobin in the nucleus responds to Wnt signaling

When plakoglobin is present in desmosomes, it interacts with desmoglein and desmocollin, and when in adherens junctions it interacts with E-cadherin in the cytoplasmic component [15]. Recently, additional evidence has suggested that plakoglobin contributes a low level of transcriptional activity to the Wnt signal transduction cascade in the nucleus $[19,20]$. Although, it has been confirmed that SOX4 modulates Wnt signaling via interaction with $\beta$-catenin [5,23], the role of plakoglobin in Wnt signaling is still debated. To investigate where and under what conditions SOX4 and plakoglobin interact with each other, we used confocal microscopy to determine whether we could observe subcellular colocalization of HA-SOX4 and plakoglobin (Figure 2). After treatment of PC3M cells that stably-expressed HA-SOX4 with recombinant human WNT3A, we observed that the interaction between HA-SOX4 and plakoglobin was slightly increased in the nucleus. In contrast, this was not observed in the non-transfected adjacent cells. We observed the same phenomenon in the LNCaP-HA-SOX4 stable cell line (Additional file 1 Figure S1). Furthermore, when we treated with nuclear export inhibitor, leptomycin b (LMB) to inhibit nuclear export of SOX4 and plakoglobin, the co-localization was significantly increased compared to treating the cells with either WNT3A or LMB alone (Figure 2). To confirm these enhanced interactions, we performed co- 


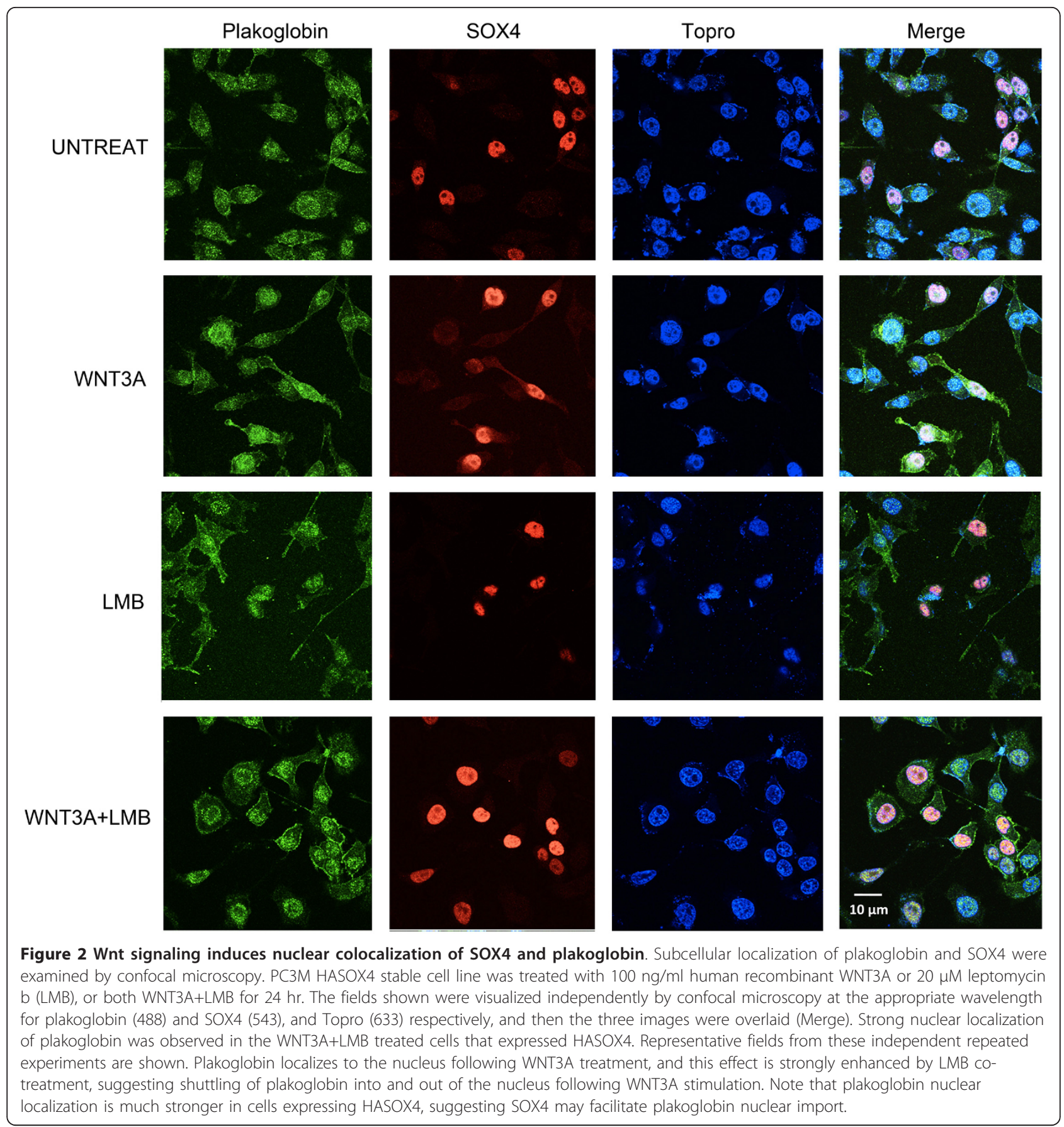

immunoprecipitation under the same Wnt-induced conditions in LNCaP-HA-SOX4 cells (Figure 3A). The interaction in the whole cell lysate was quantified and significantly increased when we treated with WNT3A and LMB together (Figure 3B). In addition, to determine that SOX4 and plakoglobin interact in the nucleus, we prepared cytosolic and nuclear fractions to confirm the subcellular interaction (Figure 3C). Immunoblots against
AKT and nuclear lamin were used as controls to demonstrate the purity of the nuclear and cytosolic fractions (Figure 3C). The quantitative results showed that the interaction in the nuclear but not cytosolic fraction was significantly increased in response to the WNT3A and LMB treatment (Figure 3D). These results show that SOX4 and plakoglobin physically interact in the nucleus of LNCaP cells. 


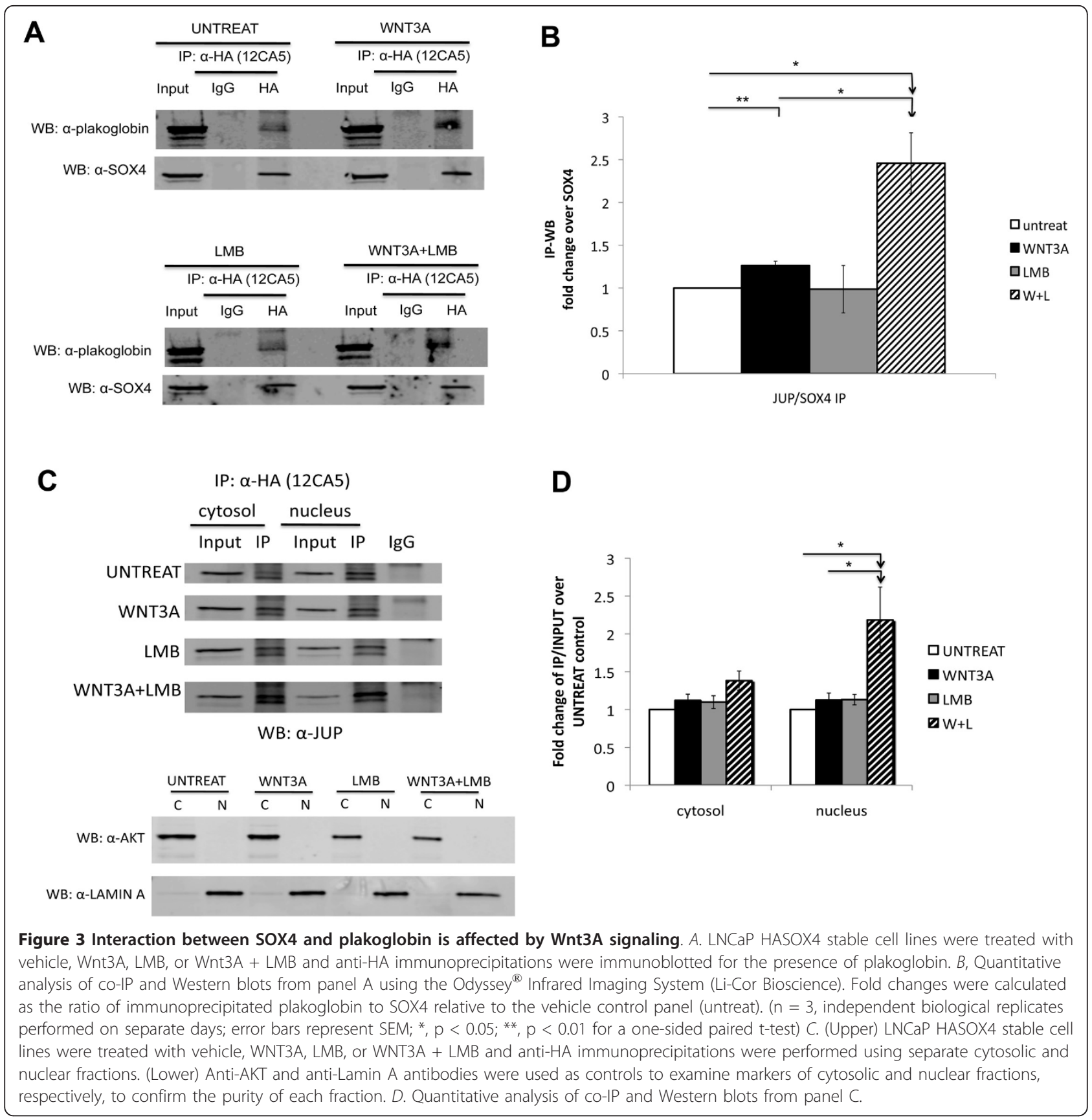

Wnt target genes and SOX4-target genes are affected by SOX4-plakoglobin interaction

To address the functional consequences of modulation of SOX4 transcriptional activity by the SOX4-plakoglobin complex, we tested expression of several genes including the Wnt target gene AXIN2 [29], as well as SOX4 targets DICER1 and DHX9 [23]. To characterize if SOX4 DNA binding activity is changed by Wntinduced interaction with plakoglobin, we performed ChIP assays for AXIN2, DICER1, DHX9, and SOX4 (Figure $4 \mathrm{~A}, \mathrm{~B})$. Compared to untreated LNCaP HASOX4 cells, the binding of SOX4 to AXIN2, DICER1, and $D H X 9$ promoters was increased after Wnt signaling was induced. However, SOX4 binding was decreased after treatment with both WNT3A and LMB. This difference indicates that increasing the interaction between SOX4 and plakoglobin could inhibit SOX4 binding activity to downstream targets and may inhibit SOX4 transcriptional activity. Quantitative realtime-PCR (qPCR) analysis of the LNCaP SOX4 stable cell line showed reductions in AXIN2, SOX4, and DHX9 upon co-treatment with WNT3A and LMB (Figure 4C). To determine 


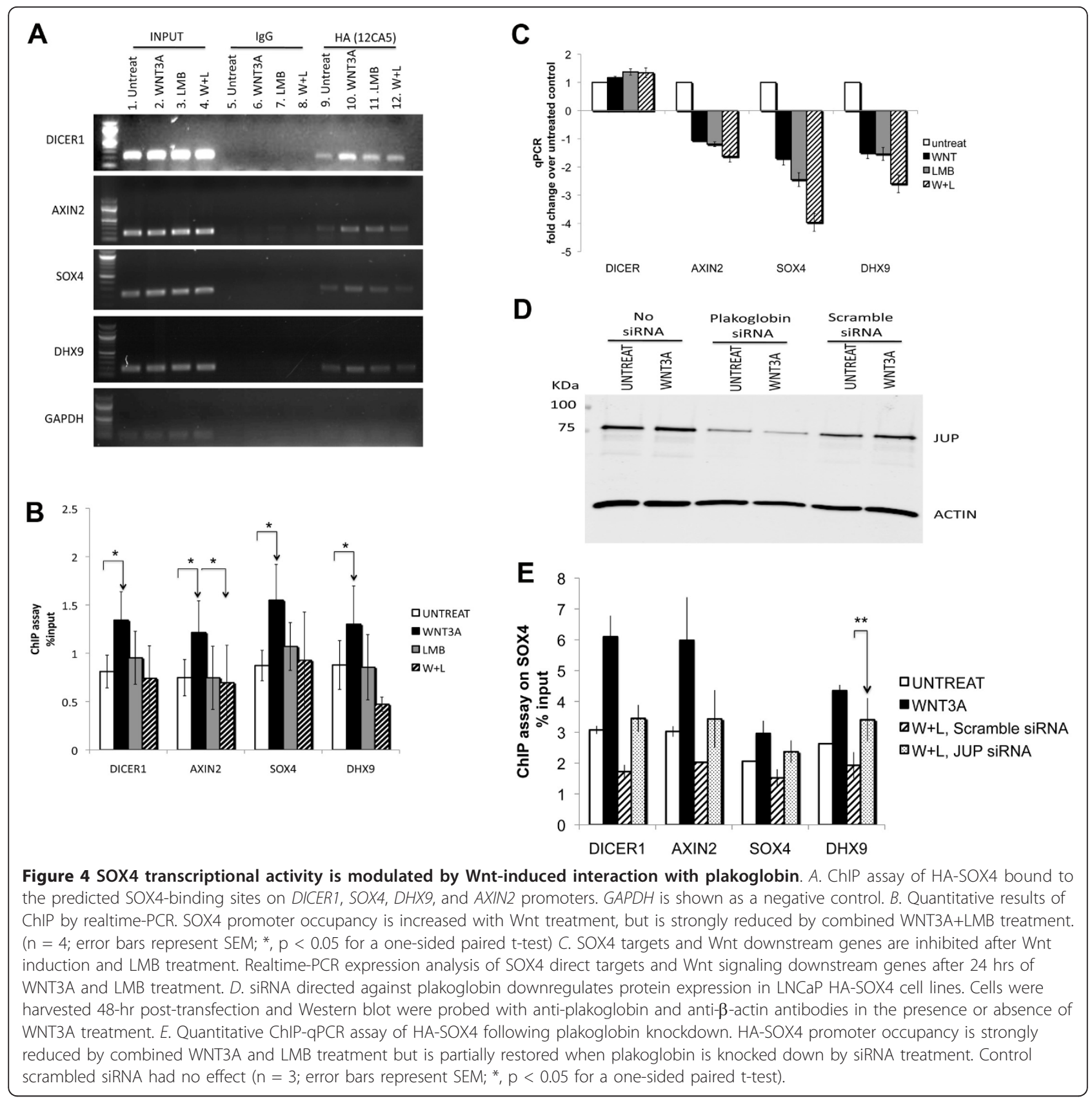

whether the effects from combined WNT3A and LMB treatment were dependent on plakoglobin, we targeted plakoglobin by siRNA to determine whether reduced plakoglobin levels could rescue the effects we observed on SOX4 binding to target promoters. Transfection of LNCaP HA-SOX4 cells with plakoglobin siRNA or scrambled control siRNA resulted in approximately 50\% knockdown of endogenous plakoglobin protein levels (Figure 4D). Furthermore, plakoglobin siRNA partially rescued SOX4 binding to target promoters by ChIP assay in the presence of WNT3A and LMB, while scrambled siRNA had no effect (Figure 4E). These data suggest that SOX4-plakoglobin interactions may interfere with SOX4-mediated transcription in response to Wnt signaling due to reduced promoter occupancy.

\section{SOX4-plakoglobin complex modulates $\beta$-catenin-mediated} transcriptional activity

To evaluate whether SOX4-plakoglobin complex affects the transcriptional activity of $\beta$-catenin, we performed luciferase reporter assays with $\mathrm{T}$ cell factor (TCF) reporter plasmids containing wild type TCF binding sites 
(TOP-flash) or mutated TCF binding site (FOP-flash) [30] (Figure 5A). As expected, the TCF/ $\beta$-catenin luciferase reporter was significantly increased after we induced Wnt signaling with recombinant WNT3A when compared to untreated cells. In contrast, co-treatment with WNT3A and LMB, strongly inhibited increases in luciferase activity back to baseline unstimulated levels, suggesting that WNT3A+ LMB-induced SOX4-plakoglobin complexes could compete with and inhibit the transcriptional activity of $\beta$-catenin. In addition, we performed anti- $\beta$-catenin ChIP assays on the AXIN2, $c$ $M y c$, and $D K K 1$ promoters, and observed that occupancy of these promoters was stimulated by WNT3A, but that stimulation was inhibited by co-treatment with WNT3A and LMB (Figure 5B). These results suggest that $\beta$-catenin activity was affected by the SOX4-

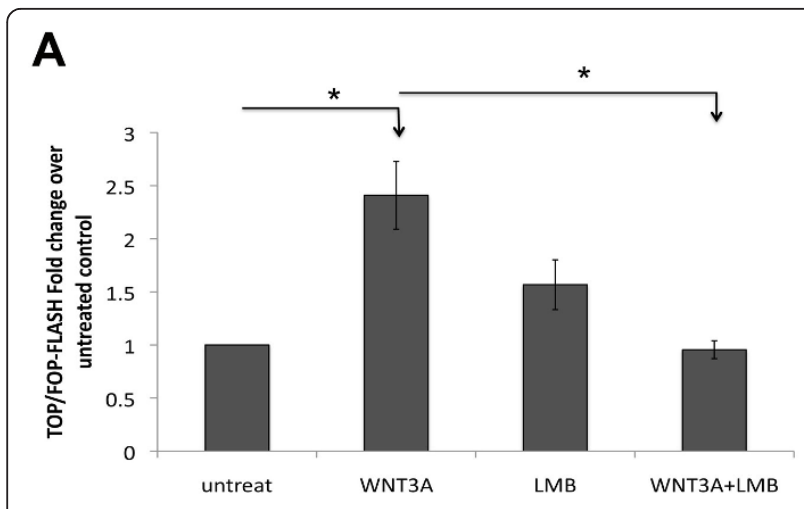

B

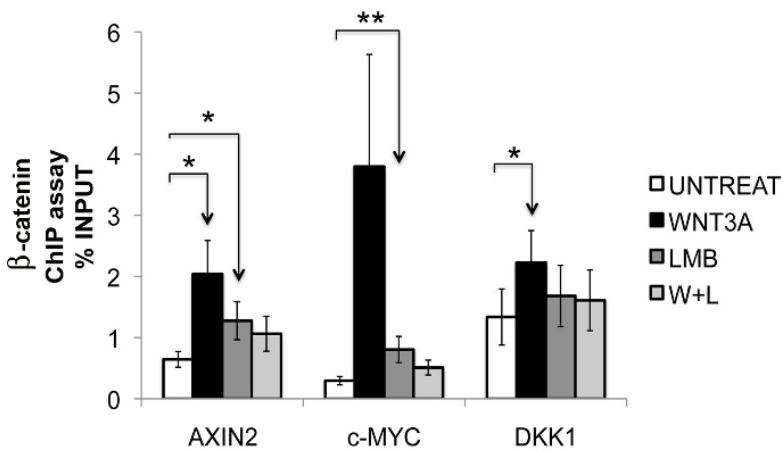

Figure 5 Wnt signaling is downregulated by SOX4-plakoglobin complex. A. Luciferase assay of LNCaP HASOX4 stable cell line cotransfected with $40 \mathrm{ng}$ of TK-Renilla with either $500 \mathrm{ng}$ of TOP-flash or FOP-flash plasmids. Luciferase activity was increased after Wnt inducement, but decreased in combined WNT3A+LMB treatment. (n $=3$; error bars represent SEM; ${ }^{*}, \mathrm{p}<0.05$ for a one-sided paired ttest) B. Quantitative results of ChIP of $\beta$-catenin by realtime-PCR. $\beta$ catenin promoter occupancies of Wnt downstream targets, AXIN2, $c$ $M Y C$, and DKK1, were increased with Wht treatment, but is strongly reduced by combined WNT3A+LMB treatment. ( $n=3$; error bars represent SEM; ${ }^{*}, \mathrm{p}<0.05 ;{ }^{* *}, \mathrm{p}<0.01$ for a one-sided paired t-test) plakoglobin complex and that plakoglobin may compete with $\beta$-catenin binding to SOX4 and/or TCF/LEF in the nucleus.

\section{Discussion}

While SOX4 expression is elevated in many malignancies and is tightly correlated with prostate cancer tumor grade, little is known of the mechanism by which SOX4 affects the progression of prostate cancer. Using LCMS/MS proteomic analysis, we identified a novel SOX4 binding protein, junction plakoglobin, in $\mathrm{LNCaP}$ prostate cancer cells. We observed a physical association between SOX4 and plakoglobin with both epitopetagged and endogenous SOX4. Although the interactions of SOX4 with plakoglobin were enhanced by cotreatment of WNT3A and LMB, we could detect this interaction using four different untransfected and unstimulated cell types (Figure 1F-I) treated with DNase I. In addition, confocal microscopy and co-immunoprecipitation demonstrated co-localization of SOX4 and plakoglobin in the nucleus when Wnt signaling was induced. ChIP assays showed the SOX4-plakoglobin complex affected SOX4 DNA binding activity to the AXIN2, DICER 1, and DHX9 promoters that are Wnt signaling downstream genes and SOX4-associated targets. In addition, mRNA expression changes were detected in AXIN2, DICER1, and DHX9 by realtime-PCR. These data suggest that the SOX4-plakoglobin complex may inhibit Wnt signaling. Indeed, conditions that induced the increased interaction between SOX4 and plakoglobin caused $\beta$-catenin TOP-FLASH transcriptional activity to be downregulated and reduced occupancy of the $c-M y c$ promoter by $\beta$-catenin.

It is known that SOX4 can directly interact with $\beta$ catenin to enhance Wnt signaling [5,23], but mechanistic data remain very limited. We found that SOX4 interacts with plakoglobin in a WNT3A-dependent manner in our experimental cancer model. Our model not only supports the hypothesis that SOX4 may stabilize $\beta$-catenin [5], but also suggests a model in which SOX4 can modulate Wnt signaling by binding either $\beta$-catenin or plakoglobin (Figure 6). In this model, transcriptional responses to Wnt signaling are increased by SOX4- $\beta$ catenin interactions, and subsequently reduced by SOX4-plakoglobin interactions that facilitate nuclear export of SOX4. However, when nuclear export is inhibited, plakoglobin competes with $\beta$-catenin for binding to SOX4 and TCF/LEF factors, downregulating Wntresponsive transcription and reducing SOX4-DNA binding. This model is supported by the observation that combination treatment with WNT3A and LMB enhanced SOX4-plakoglobin interactions, reduced TCF/ $\beta$-catenin TOP-FLASH transcription, reduced SOX4DNA binding and $\beta$-catenin DNA binding in ChIP 


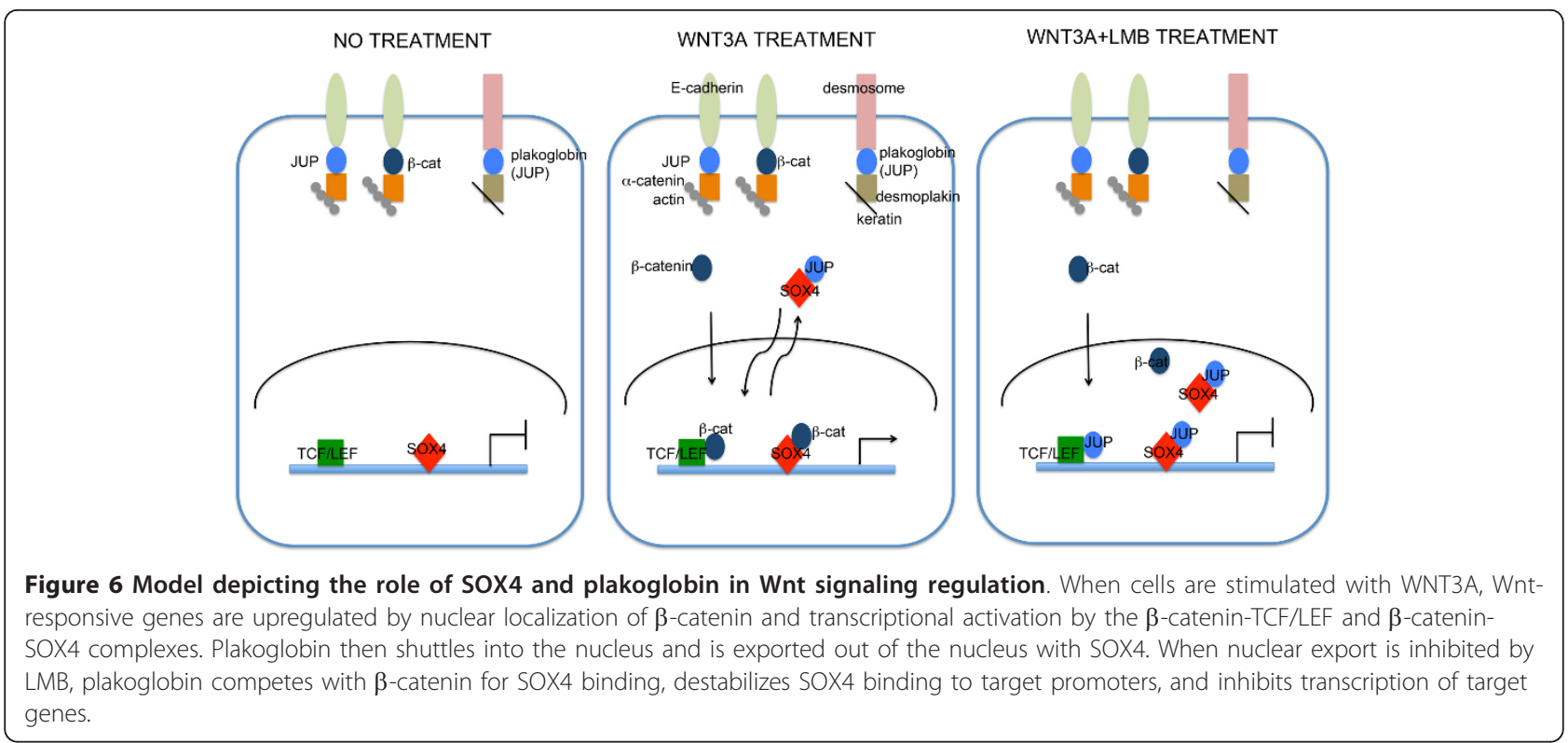

assays, and reduced expression of SOX4 downstream targets (Figures 4 and 5). Consistent with our model, plakoglobin shows little transcriptional activity compared to $\beta$-catenin in cell lines that lack $\beta$-catenin [19].

Our confocal data show that plakoglobin nuclear localization is strongly enhanced by LMB co-treatment, suggesting shuttling of plakoglobin into and out of the nucleus following WNT3A stimulation. Although LMB is an artificial stimulus, others have shown that plakoglobin overexpression can lead to nuclear localization [31,32], and plakoglobin is overexpressed [33] and amplified [34] in several types of cancer. Moreover, plakoglobin nuclear localization was much stronger in cells expressing HASOX4, suggesting cytoplasmic SOX4 may facilitate plakoglobin nuclear import. Thus, SOX4 may induce nuclear import of plakoglobin in response to WNT3A while plakoglobin destabilizes SOX4 from DNA binding, facilitating nuclear export of SOX4. It is as yet unclear what signals or modifications might tip the balance between nuclear import and export of plakoglobin-SOX4 complexes, or whether shuttling might be constitutive.

The role of plakoglobin during cancer progression is still controversial. When plakoglobin is overexpressed, it induces cell migration and mobility in HCT116 cells, suggesting that plakoglobin may have some oncogenic effects [31]. In contrast, several reports have demonstrated that plakoglobin has a tumor-suppressive effect that inhibits tumor cell growth $[27,35]$. The tumor suppressive activity of plakoglobin may be via nuclear translocation to antogonize $\beta$-catenin binding to TCF/LEF proteins in keratinocytes [36], supporting our model that plakoglobin competes with SOX4- $\beta$-catenin interactions in the nucleus.

\section{Conclusion}

In summary, we have demonstrated that SOX4 interacts with plakoglobin in a Wnt-dependent manner in LNCaP cells and that this complex may function to inhibit Wnt signaling. Additional studies will be required to elucidate the detailed mechanisms by which SOX4-plakoglobin interactions may affect Wnt signaling, but the role of the SOX4-plakoglobin complex provides novel insights into the role of SOX4 in Wnt signaling and prostate cancer progression.

\section{Additional material}

Additional file 1: Figure.S1 Wnt signaling induces nuclear colocalization of SOX4 and plakoglobin. Subcellular localization of plakoglobin and SOX4 were examined by confocal microscopy. LNCaP HASOX4 stable cell line was treated with $100 \mathrm{ng} / \mathrm{ml}$ human recombinant WNT3A or $20 \mu \mathrm{M}$ leptomycin b (LMB), or both WNT3A+LMB for $24 \mathrm{hr}$. The fields shown were visualized independently by confocal microscopy at the appropriate wavelength for plakoglobin (488) and SOX4 (543), and Topro (633) respectively, and then the three images were overlaid (Merge). Strong nuclear localization of plakoglobin was observed in the WNT3A+LMB treated cells that expressed HASOX4. Representative fields from these independent repeated experiments are shown. Plakoglobin localizes to the nucleus following WNT3A treatment, and this effect is strongly enhanced by LMB co-treatment, suggesting shuttling of plakoglobin into and out of the nucleus following WNT3A stimulation. Note that plakoglobin nuclear localization is much stronger in cells expressing HASOX4, suggesting SOX4 may facilitate plakoglobin nuclear import. 


\section{Acknowledgements}

We thank Dr. Tamara Caspary for advice on experimental conditions for LMB treatments, Dr. Chris Scharer for advice on SOX4 binding site analysis, Dr. Cornel Phillip for advice with WNT3A treatment, and Dr. Andrew Kowalczyk for providing primary human keratinocytes. This work was supported by National Cancer Institute grants R01CA106826 (C.S.M.) and P30CA138292 (J. P.).

\section{Author details}

'Program in Genetics \& Molecular Biology, Emory University, Atlanta, GA 30322, USA. ²Department of Pathology \& Laboratory Medicine, Emory University School of Medicine, Atlanta, GA 30322, USA. ${ }^{3}$ Department of Human Genetics, Emory Proteomics Service Center, Emory University, Atlanta, GA 30322, USA. "Winship Cancer Institute, Emory University School of Medicine, Atlanta, GA 30322, USA. ${ }^{5}$ Department of Structural Biology, St. Jude Proteomics Facility, St. Jude Children's Research Hospital, Memphis, TN 38105, USA. ${ }^{6}$ Department of Developmental Neurobiology, St. Jude Proteomics Facility, St. Jude Children's Research Hospital, Memphis, TN 38105, USA.

\section{Authors' contributions}

YHL performed the immunopurifications of SOX4, the ChIP assays, co-IPS, luciferase assays, and helped write the manuscript. JC cloned SOX4 into the pREP4-BLRPwt-IRES-BirA-XL9 plasmid and performed co-IPS. DC performed the proteomics analysis. MEF and KDB performed immunofluorescence confocal microscopy. JP advised on protein purification, supervised the proteomics analysis, and edited the manuscript. AN supervised the confocal microscopy and edited the manuscript. CSM conceived the study, participated in its design and coordination, and co-wrote the manuscript. All authors read and approved the final manuscript.

Received: 27 May 2011 Accepted: 19 November 2011 Published: 19 November 2011

\section{References}

1. Schepers GE, Teasdale RD, Koopman P: Twenty pairs of sox: extent, homology, and nomenclature of the mouse and human sox transcription factor gene families. Dev Cell 2002, 3(2):167-170.

2. Akiyama $H$, Lyons JP, Mori-Akiyama $Y$, Yang $X$, Zhang $R$, Zhang Z, Deng JM, Taketo MM, Nakamura T, Behringer RR, McCrea PD, de Crombrugghe B: Interactions between Sox 9 and beta-catenin control chondrocyte differentiation. Genes Dev 2004, 18(9):1072-1087.

3. Zorn AM, Barish GD, Williams BO, Lavender P, Klymkowsky MW, Varmus HE: Regulation of Wnt signaling by Sox proteins: XSox17 alpha/beta and XSox3 physically interact with beta-catenin. Mol Cell 1999, 4(4):487-498.

4. Takash W, Canizares J, Bonneaud N, Poulat F, Mattei MG, Jay P, Berta P: SOX7 transcription factor: sequence, chromosomal localisation, expression, transactivation and interference with Wnt signalling. Nucleic Acids Res 2001, 29(21):4274-4283.

5. Sinner D, Kordich JJ, Spence JR, Opoka R, Rankin S, Lin SC, Jonatan D, Zorn AM, Wells JM: Sox17 and Sox4 differentially regulate beta-catenin/Tcell factor activity and proliferation of colon carcinoma cells. Mol Cell Biol 2007, 27(22):7802-7815.

6. Look AT: Oncogenic transcription factors in the human acute leukemias. Science 1997, 278(5340):1059-1064.

7. Aaboe M, Birkenkamp-Demtroder K, Wiuf C, Sorensen FB, Thykjaer T, Sauter G, Jensen KM, Dyrskjot L, Orntoft T: SOX4 expression in bladder carcinoma: clinical aspects and in vitro functional characterization. Cancer Res 2006, 66(7):3434-3442.

8. Liu P, Ramachandran S, Ali Seyed M, Scharer CD, Laycock N, Dalton WB, Williams H, Karanam S, Datta MW, Jaye DL, Moreno CS: Sex-determining region $\mathrm{Y}$ box 4 is a transforming oncogene in human prostate cancer cells. Cancer Res 2006, 66(8):4011-4019.

9. Lee CJ, Appleby VJ, Orme AT, Chan WI, Scotting PJ: Differential expression of SOX4 and SOX11 in medulloblastoma. J Neurooncol 2002, 57(3):201-214

10. Rhodes DR, Yu J, Shanker K, Deshpande N, Varambally R, Ghosh D, Barrette T, Pandey A, Chinnaiyan AM: Large-scale meta-analysis of cancer microarray data identifies common transcriptional profiles of neoplastic transformation and progression. Proc Natl Acad Sci USA 2004, 101(25):9309-9314.
11. Huang WY, Yue L, Qiu WS, Wang LW, Zhou XH, Sun YJ: Prognostic value of CRM1 in pancreas cancer. Clin Invest Med 2009, 32(6):E315.

12. Suzuki T, Shen H, Akagi K, Morse HC, Malley JD, Naiman DQ, Jenkins NA, Copeland NG: New genes involved in cancer identified by retroviral tagging. Nat Genet 2002, 32(1):166-174.

13. Lund AH, Turner G, Trubetskoy A, Verhoeven E, Wientjens E, Hulsman D, Russell R, DePinho RA, Lenz J, van Lohuizen M: Genome-wide retroviral insertional tagging of genes involved in cancer in Cdkn2a-deficient mice. Nat Genet 2002, 32(1):160-165.

14. Shin MS, Fredrickson TN, Hartley JW, Suzuki T, Agaki K, Morse HC: Highthroughput retroviral tagging for identification of genes involved in initiation and progression of mouse splenic marginal zone lymphomas. Cancer Res 2004, 64(13):4419-4427.

15. Cowin P, Kapprell HP, Franke WW, Tamkun J, Hynes RO: Plakoglobin: a protein common to different kinds of intercellular adhering junctions. Cell 1986, 46(7):1063-1073.

16. Peifer $M$, Wieschaus $E$ : The segment polarity gene armadillo encodes a functionally modular protein that is the Drosophila homolog of human plakoglobin. Cell 1990, 63(6):1167-1176.

17. McCrea PD, Turck CW, Gumbiner B: A homolog of the armadillo protein in Drosophila (plakoglobin) associated with E-cadherin. Science 1991 254(5036):1359-1361

18. Aberle H, Butz S, Stappert J, Weissig H, Kemler R, Hoschuetzky H: Assembly of the cadherin-catenin complex in vitro with recombinant proteins. $J$ Cell Sci 1994, 107(Pt 12):3655-3663.

19. Maeda O, Usami N, Kondo M, Takahashi M, Goto H, Shimokata K, Kusugami K, Sekido Y: Plakoglobin (gamma-catenin) has TCF/LEF familydependent transcriptional activity in beta-catenin-deficient cell line. Oncogene 2004, 23(4):964-972.

20. Shimizu M, Fukunaga $Y$, Ikenouchi J, Nagafuchi A: Defining the roles of beta-catenin and plakoglobin in LEF/T-cell factor-dependent transcription using beta-catenin/plakoglobin-null F9 cells. Mol Cell BiO 2008, 28(2):825-835.

21. Xu P, Duong DM, Peng J: Systematical optimization of reverse-phase chromatography for shotgun proteomics. Journal of proteome research 2009, 8(8):3944-3950.

22. Ramachandran S, Liu P, Young AN, Yin-Goen Q, Lim SD, Laycock N, Amin MB, Carney JK, Marshall FF, Petros JA, Moreno CS: Loss of HOXC6 Expression Induces Apoptosis in Prostate Cancer Cells. Oncogene 2005, 24(1):188-198.

23. Scharer CD, McCabe CD, Ali-Seyed M, Berger MF, Bulyk ML, Moreno CS: Genome-wide promoter analysis of the SOX4 transcriptional network in prostate cancer cells. Cancer Res 2009, 69(2):709-717.

24. Holden $\mathrm{P}$, Horton WA: Crude subcellular fractionation of cultured mammalian cell lines. BMC Res Notes 2009, 2:243.

25. Livak KJ, Schmittgen TD: Analysis of relative gene expression data using real-time quantitative PCR and the 2(-Delta Delta C(T)) Method. Methods 2001, 25(4):402-408.

26. Odom DT, Zizlsperger N, Gordon DB, Bell GW, Rinaldi NJ, Murray HL, Volkert TL, Schreiber J, Rolfe PA, Gifford DK, Fraenkel E, Bell Gl, Young RA: Control of pancreas and liver gene expression by HNF transcription factors. Science 2004, 303(5662):1378-1381.

27. Rieger-Christ KM, Ng L, Hanley RS, Durrani O, Ma H, Yee AS, Libertino JA, Summerhayes IC: Restoration of plakoglobin expression in bladder carcinoma cell lines suppresses cell migration and tumorigenic potential. Br J Cancer 2005, 92(12):2153-2159.

28. Barker DF, Campbell AM: The birA gene of Escherichia coli encodes a biotin holoenzyme synthetase. J Mol Biol 1981, 146(4):451-467.

29. Jho EH, Zhang T, Domon C, Joo CK, Freund JN, Costantini F: Wnt/betacatenin/Tcf signaling induces the transcription of Axin2, a negative regulator of the signaling pathway. Mol Cell Biol 2002, 22(4):1172-1183.

30. Korinek V, Barker N, Morin PJ, van Wichen D, de Weger R, Kinzler KW, Vogelstein B, Clevers H: Constitutive transcriptional activation by a betacatenin-Tcf complex in APC-/- colon carcinoma. Science 1997, 275(5307):1784-1787.

31. Pan H, Gao F, Papageorgis $P$, Abdolmaleky HM, Faller DV, Thiagalingam S: Aberrant activation of gamma-catenin promotes genomic instability and oncogenic effects during tumor progression. Cancer Biol Ther 2007, 6(10):1638-1643

32. Kolligs FT, Kolligs B, Hajra KM, Hu G, Tani M, Cho KR, Fearon ER: gammacatenin is regulated by the APC tumor suppressor and its oncogenic 
activity is distinct from that of beta-catenin. Genes Dev 2000,

14(11):1319-1331.

33. Davidson B, Berner A, Nesland JM, Risberg B, Berner HS, Trope CG,

Kristensen GB, Bryne M, Ann Florenes V: E-cadherin and alpha-, beta-, and gamma-catenin protein expression is up-regulated in ovarian carcinoma cells in serous effusions. J Pathol 2000, 192(4):460-469.

34. Varis A, Wolf M, Monni O, Vakkari ML, Kokkola A, Moskaluk C, Frierson H, Powell SM, Knuutila S, Kallioniemi A, El-Rifai W: Targets of gene amplification and overexpression at $17 \mathrm{q}$ in gastric cancer. Cancer Res 2002, 62(9):2625-2629.

35. Winn RA, Bremnes RM, Bemis L, Franklin WA, Miller YE, Cool C, Heasley LE: gamma-Catenin expression is reduced or absent in a subset of human lung cancers and re-expression inhibits transformed cell growth. Oncogene 2002, 21(49):7497-7506.

36. Hu P, Berkowitz P, O'Keefe EJ, Rubenstein DS: Keratinocyte adherens junctions initiate nuclear signaling by translocation of plakoglobin from the membrane to the nucleus. J Invest Dermatol 2003, 121(2):242-251.

doi:10.1186/1471-2121-12-50

Cite this article as: Lai et al: SOX4 interacts with plakoglobin in a

Wnt3a-dependent manner in prostate cancer cells. BMC Cell Biology 2011 12:50.

\section{Submit your next manuscript to BioMed Central} and take full advantage of:

- Convenient online submission

- Thorough peer review

- No space constraints or color figure charges

- Immediate publication on acceptance

- Inclusion in PubMed, CAS, Scopus and Google Scholar

- Research which is freely available for redistribution

Submit your manuscript at www.biomedcentral.com/submit 